This paper was published in Applied Optics and is made available as an electronic reprint with the

permission of OSA. The paper can be found at the following URL on the OSA website:

http: //www.opticsinfobase.org/abstract.cfm?URI=ao-46-29-7218

Systematic or multiple reproduction or distribution to multiple locations via electronic or other means is prohibited and is subject to penalties under law.

\title{
Turbulence-induced fading probability in coherent optical communication through the atmosphere
}

\author{
N. Perlot \\ Institute of Communication and Navigation, DLR Oberpfaffenhofen, 82234 Wessling, Germany \\ nicolas.perlot@dlr.de
}

Received 2 April 2007; revised 6 August 2007; accepted 10 August 2007; posted 15 August 2007 (Doc. ID 81719); published 5 October 2007

\begin{abstract}
To assess the coherent detection of an optical signal perturbed by atmospheric turbulence, the loss in the mean signal-to-noise ratio (SNR) is usually invoked although it constitutes a limited description of the signal fluctuations. To produce statistical distributions of the SNR, we generate random optical fields. A $5 / 3$-power law for the phase structure function is considered. The benefit of a wavefront tilt correction is assessed. Based on the 1\%-probability fade, an optimum receiver size is found. For phase fluctuations only, a similarity between the signal distribution and the beta distribution is observed. Phase and amplitude are assumed independent, and the influence of amplitude perturbations is assessed with a scintillation index of 2 . Turbulence impairments are compared for a coherent receiver and a directdetection receiver. (C) 2007 Optical Society of America
\end{abstract}

OCIS codes: $\quad 010.1300,010.1330,060.1660,060.4510$.

\section{Introduction}

When an optical signal is transmitted, there exist several reasons for using a coherent detection. Compared to its incoherent counterpart, a coherent receiver is generally more sensitive to the signal and less sensitive to background light. However, when the signal is transmitted through the atmosphere, optical turbulence randomly distorts the phase and the amplitude of the wave. Signal fades associated with optical turbulence strongly depend on the detection type. Unlike direct detection, coherent detection is impaired by phase distortions, which set a limit on the effective receiver size.

Much work has been done on the characterization of scintillation and on its impact on direct-detection systems $[1,2]$. For coherent systems, the performance evaluation is generally based on the mean signal-tonoise ratio (SNR) characterized by Fried in 1967 $[1,3,4]$. This long-term SNR does not, however, allow predictions regarding signal fading probabilities. The investigation of the heterodyne signal distribution started more than three decades ago. Churnside and McIntyre derived analytically an approximate distri-

0003-6935/07/297218-09\$15.00/0

(C) 2007 Optical Society of America bution for a limited range of the receiving aperture size $[5,6]$. The analytical derivation of the coherentsignal distribution is difficult, even when the statistics of the optical field are known. The reason is the spatial averaging operated by the receiving aperture on the optical field, which generally makes expressions of the signal statistics intractable. Distributions computed from the generation of correlated phase samples in the receiving aperture were also reported $[7,8]$. There is still, however, no accurate description of the signal distribution as a function of the aperture size. Increasing the aperture size reduces scintillation but also the heterodyne efficiency. Recently, there has been a regain of interest in optical freespace coherent communications with the fabrication of terminals implementing a homodyne binary phase shift keying (BPSK) $[9,10]$. To use this technology through the atmosphere, a precise prediction of the turbulence influence is required.

Here, we also resort to simulations. Spatial realizations of the optical field in the receiving-aperture plane are produced. Associated SNR statistics are analyzed as a function of the aperture size. As a possible improvement through adaptive optics, we consider the tilt correction. The paper is structured as follows. First, we define the detected signal photocurrent and express it in terms of the perturbed optical 
field. In Section 3, the SNR distribution is studied under phase perturbations only. Section 4 deals with the additional effect of amplitude perturbations (scintillation). Under scintillation, a brief comparison of impairments is made with direct detection. The paper concludes with Section 5.

\section{Signal Definition}

A coherent detection is obtained by adding a local oscillator (LO) to the received field on the photodetector. Because optical turbulence has a negligible depolarization effect [11], we assume a polarization match of the two fields on the detector and consider scalar fields. The analysis of the photocurrent resulting from the detection can be done by considering the combined fields either in the focal plane or in the aperture plane [3]. In the aperture plane, the photocurrent term $i$ containing the interference between the scalar LO field $E_{\mathrm{LO}}$ and the scalar signal field $E_{s}$ is given by [3]:

$$
i=\operatorname{Re}\left[\int W(\mathbf{r}) E_{s}(\mathbf{r}) E_{\mathrm{LO}} *(\mathbf{r}) \mathrm{d} \mathbf{r}\right],
$$

where Re denotes the real part, $\mathbf{r}$ is a twodimensional vector in the aperture plane, and $W(\mathbf{r})$ is the aperture window function. Depending on the definition of the irradiance, Eq. (1) may vary by a constant factor. To simplify the analysis, we assume that the LO as well as the unperturbed signal field are monochromatic plane waves over the aperture and that the LO phase maximizes the current $i$ at any time. This amounts to considering a receiver with a perfect optical phase-locked loop assuring a homodyne detection. Temporal spectra of turbulenceinduced wavefront perturbations can be found in [12]. Setting $E_{\mathrm{LO}}(\mathbf{r})=1$, Eq. (1) yields

$$
\begin{aligned}
i & =\left|\int W(\mathbf{r}) E_{s}(\mathbf{r}) \mathrm{d} \mathbf{r}\right| \\
& =\left|\int W(\mathbf{r}) A(\mathbf{r}) \exp [j \phi(\mathbf{r})] \mathrm{d} \mathbf{r}\right|,
\end{aligned}
$$

where $A(\mathbf{r})$ and $\phi(\mathbf{r})$, respectively, are the perturbed amplitude and phase of the optical signal, and $j=\sqrt{-1}$. We assume $A(\mathbf{r})$ and $\phi(\mathbf{r})$ are statistically homogeneous over the aperture and we normalize the amplitude according to

$$
\left\langle A^{2}\right\rangle=\langle I\rangle=1
$$

where $\langle\cdot\rangle$ denotes ensemble averaging and $I$ is the optical field intensity. Furthermore, we consider a circular aperture of diameter $D$ and normalize the aperture window function over the aperture area:

$$
W(\mathbf{r})= \begin{cases}4 /\left(\pi D^{2}\right), & \text { if }|\mathbf{r}| \leq D / 2 \\ 0, & \text { if }|\mathbf{r}|>D / 2\end{cases}
$$

The photocurrent $i$ will thus be analyzed as a dimensionless quantity. An important point underlying the following analysis is the separation of two types of variations: turbulence-induced signal variations and receiver noise variations. Turbulence-induced fluctuations are on the order of kilohertz, whereas the receiver noise bandwidth for a common optical transmission is on the order of gigahertz. Because turbulent perturbations are slow compared to a bit duration, we can consider a turbulence-induced probability distribution for the SNR. This distribution is directly related to that of the signal photocurrent $i$.

\section{Phase Perturbations}

\section{A. Mean Signal-to-Noise Ratio}

We consider the phase as a Gaussian field characterized by the following spatial structure function,

$$
D_{\phi}(\rho)=6.88\left(\frac{\rho}{r_{\phi}}\right)^{5 / 3},
$$

where $\rho$ is the module of the variable vector $\rho$ in the aperture plane and $r_{\phi}$ is a characteristic length. Because it defines the phase structure function, the characteristic length is noted $r_{\phi}\left(r_{0}\right.$, the so-called coherence diameter, is generally used to define the wave structure function [3]). Equation (5) is an approximation of the structure function derived from the Rytov theory: there exist more complex and more precise expressions of $D_{\phi}(\rho)$ [13]. It has been argued that phase statistics under weak fluctuations should also apply under strong fluctuations $[11,14]$. Note, however, that by considering the phase as a continuous Gaussian field, phase dislocations, which may occur under strong fluctuations [15], are not taken into account.

Without scintillation $(A=1), i$ is equal to $i_{\phi}$ defined by

$$
i_{\phi} \equiv\left|\int W(\mathbf{r}) \exp [j \phi(\mathbf{r})] \mathrm{d} \mathbf{r}\right|,
$$

and included between 0 and 1 . The mean square equals [3]:

$$
\left\langle i_{\phi}{ }^{2}\right\rangle=2 \pi \int H_{W}(\rho) R_{\phi}(\rho) \rho \mathrm{d} \rho,
$$

where $H_{W}$ is the autocorrelation function of the aperture window:

$$
H_{W}(\boldsymbol{\rho})=\int W(\mathbf{r}) W(\mathbf{r}+\boldsymbol{\rho}) \mathrm{d} \mathbf{r} .
$$


$R_{\phi}(\rho)$ is the spatial correlation function of $\exp [j \phi(\mathbf{r})]$ given by

$$
R_{\phi}(\rho)=\exp \left[-0.5 D_{\phi}(\rho)\right] .
$$

It is known [3] that, with a structure function as defined by Eq. (5), we have $\left\langle i_{\phi}{ }^{2}\right\rangle \rightarrow r_{\phi}{ }^{2} / D^{2}$ for $D / r_{\phi} \rightarrow \infty$. A well-designed coherent receiver is shotnoise limited, and the SNR is proportional to the product of $i^{2}$ with the aperture area. Because of its known asymptotic behavior, we consider the following SNR:

$$
\mathrm{SNR}_{0} \equiv\left(D / r_{\phi}\right)^{2} i^{2}
$$

Numerical realizations of the phase field $\phi(\mathbf{r})$ were generated as described in [16] with a $4 \times 4$ interpolator. Figure 1 shows $\left\langle\mathrm{SNR}_{0}\right\rangle$ in decibels $(\mathrm{dB})$ for $i=i_{\phi}$. Although $\left\langle\mathrm{SNR}_{0}\right\rangle$ is plotted versus $D / r_{\phi}$, one should consider the abscissa as a variable $D$ with respect to a constant $r_{\phi}$ (rather than a variable $r_{\phi}$ with respect to a constant $D$ ). This is because $\mathrm{SNR}_{0}$ takes into account the antenna gain, which is determined by $D^{2}$ but independent of $r_{\phi}$. The asymptotes $D^{2} / r_{\phi}{ }^{2}$ and 1 are plotted on the graph as dashed curves. Beside the case of an uncorrected wavefront, corrections of the so-called $\mathrm{Z}$ and $\mathrm{C}$ tilts are considered. The $\mathrm{Z}$ tilt corresponds to the second and third Zernike polynomials. The $\mathrm{C}$ tilt is calculated from the displacement of the centroid of the focal spot. In the absence of scintillation, the $\mathrm{C}$ tilt is equivalent to the $\mathrm{G}$ tilt, which is the average gradient of the wavefront across the aperture. The difference between $\mathrm{C}$ and $\mathrm{G}$ tilts is discussed in [17]. The difference between $\mathrm{Z}$ and $G$ tilts is discussed in [18]. From simulations, $\left\langle\mathrm{SNR}_{0}\right\rangle$ was evaluated for the three types of wavefront. Analytically, Eq. (7) was used for the nocorrection case and [19] provided expressions for the Z-tilt case. However, no analytical expression of

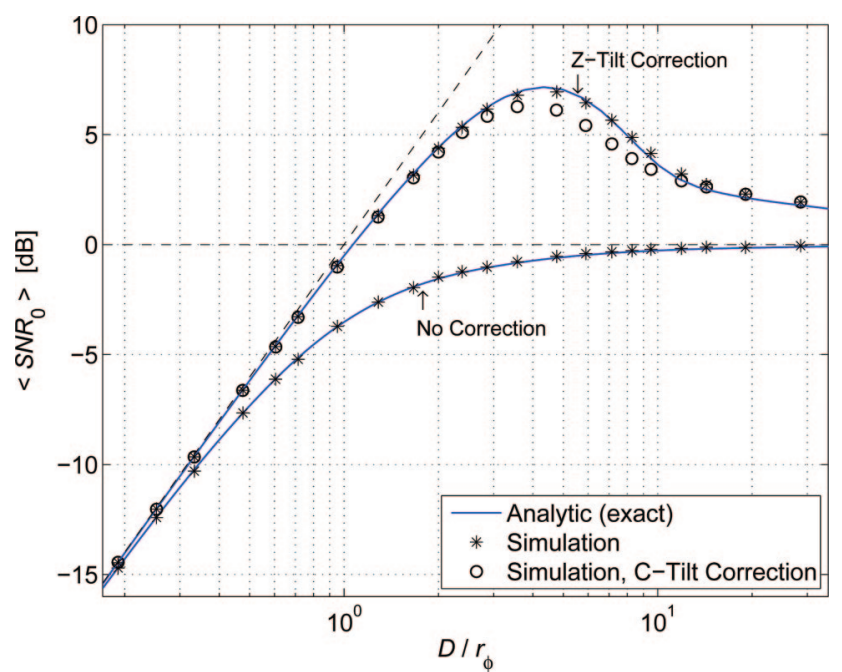

Fig. 1. (Color online) Mean $\mathrm{SNR}_{0}$ when the wavefront is not corrected and when C- and Z-tilt corrections are performed. Simulation results are compared with exact values.
$\left\langle\mathrm{SNR}_{0}\right\rangle$ for the C-tilt correction could be found. We observe in Fig. 1 a good agreement between the exact (analytical) and the simulation results. In the remaining part of the paper we drop the $\mathrm{Z}$ tilt and focus on the C-tilt correction, as it is usually the one implemented in reality.

The mean normalized SNR for the uncorrected wave, noted $\left\langle\mathrm{SNR}_{0, u}\right\rangle$, is generally approximated by

$$
\left\langle\mathrm{SNR}_{0, u}\right\rangle \cong \frac{\left(D / r_{\phi}\right)^{2}}{\left[1+\left(D / r_{\phi}\right)^{5 / 3}\right]^{6 / 5}} .
$$

For the C-tilt correction, it was found that the mean normalized SNR could be approximated by

$$
\left\langle\mathrm{SNR}_{0, C}\right\rangle \cong\left\langle\mathrm{SNR}_{0, u}\right\rangle\left(1+\frac{\left(0.985 D / r_{\phi}\right)^{1.84}}{1+\left(0.331 D / r_{\phi}\right)^{3.18}}\right) .
$$

The maximum error made by Eq. (12) is $\sim 10 \%$ compared to the simulation values.

\section{B. Signal-to-Noise Ratio Distribution}

The normalized variance of $i$ is defined by

$$
\sigma_{i, 0}^{2} \equiv \frac{\left\langle i^{2}\right\rangle}{\langle i\rangle^{2}}-1
$$

Based on simulation results, an approximation of $\sigma_{i, 0}{ }^{2}$ when $i=i_{\phi}$ can be put in the form:

$$
\sigma_{i, 0}^{2} \cong 0.273\left(\frac{\left(a D / r_{\phi}\right)^{c}}{1+\left(b D / r_{\phi}\right)^{d}}+\frac{\left(e D / r_{\phi}\right)^{f}}{1+\left(e D / r_{\phi}\right)^{f}}\right)
$$

The parameters of Eq. (14) are listed in Table 1 for the cases with and without tilt correction. Figure 2 shows $\sigma_{i, 0}^{2}$ as a function of $D / r_{\phi}$ together with the fitting curves of Eq. (14). We observe that the normalized variance of $i_{\phi}$ starts a sharp increase at $D \approx 0.5 r_{\phi}$ when there is no correction and a sharp increase at $D \approx 2 r_{\phi}$ when the tilt is corrected. The behavior of $\sigma_{i, 0}{ }^{2}$ for $D / r_{\phi} \rightarrow \infty$ can be deduced from the central-limit theorem (CLT) [20]. The integral in Eq. (6) can indeed be viewed as the infinite sum of independent optical-field cells. It results that $i$ tends to have a Rayleigh distribution and we have

$$
\lim _{D / r_{\phi} \rightarrow \infty} \sigma_{i, 0}^{2}=\frac{4}{\pi}-1
$$

Table 1. Parameter Values for Fitting Curves of $\sigma_{i, 0}{ }^{2}$

\begin{tabular}{ccc}
\hline Parameter & No Correction & C-Tilt Correction \\
\hline$a$ & 0.743 & 0.188 \\
$b$ & 0.532 & 0.180 \\
$c$ & 2.59 & 4.95 \\
$d$ & 3.30 & 5.78 \\
$e$ & 9.56 & 0.520 \\
$f$ & 22.4 & 5.22 \\
\hline
\end{tabular}




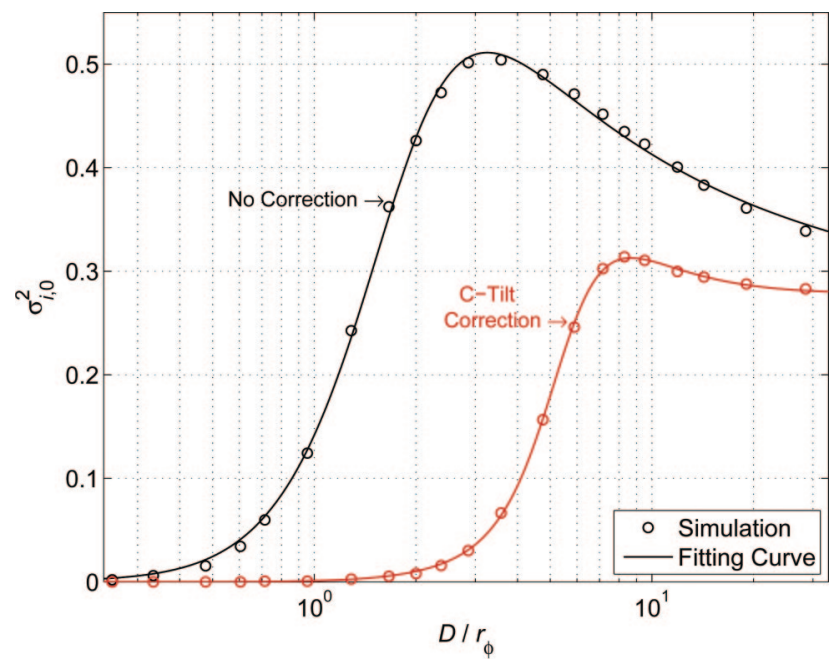

Fig. 2. (Color online) Normalized variances of $i_{\phi}$ estimated from simulations and plotted along with their fitting curves.

The factor $4 / \pi-1 \approx 0.273$ was introduced in Eq. (14) to comply with Eq. (15).

In an attempt to find a simple distribution model for $i_{\phi}$, the computed distribution of $i_{\phi}$ was compared with the beta distribution. Note that the choice of the beta distribution is based on a resemblance of the observed curves, and not on any statistical reasoning. The beta cumulated density function (CDF), noted $F_{\text {beta }}$, is given by

$$
F_{\text {beta }}(x)=\frac{B_{x}(\alpha, \beta)}{B(\alpha, \beta)}, \quad 0 \leq x \leq 1,
$$

where $B$ is the beta function, $B_{x}$ the incomplete beta function [21], $\alpha$ and $\beta$ the two distribution parameters. To assess the merit of the beta distribution, we consider, over the values taken by $x$, the maximum difference between $F_{\text {beta }}$ and the CDF $F_{i_{\phi}}$ of $i_{\phi}$. The maximum of $\left|F_{i_{\phi}}-F_{\text {beta }}\right|$ is shown in Fig. 3 as a

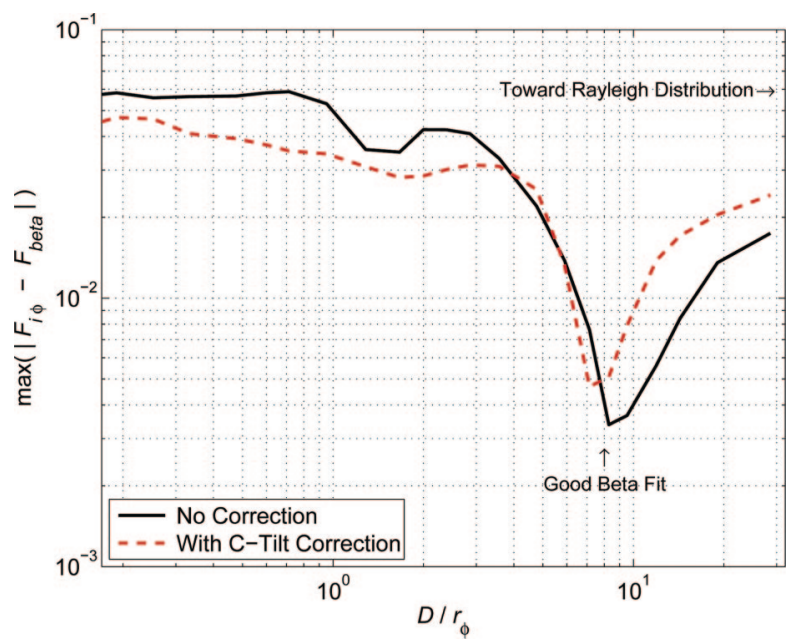

Fig. 3. (Color online) Comparison of the $i_{\phi}$ distribution with the beta distribution. The beta fit improves as $\max \left(\left|F_{i_{\phi}}-F_{\text {beta }}\right|\right)$ decreases.

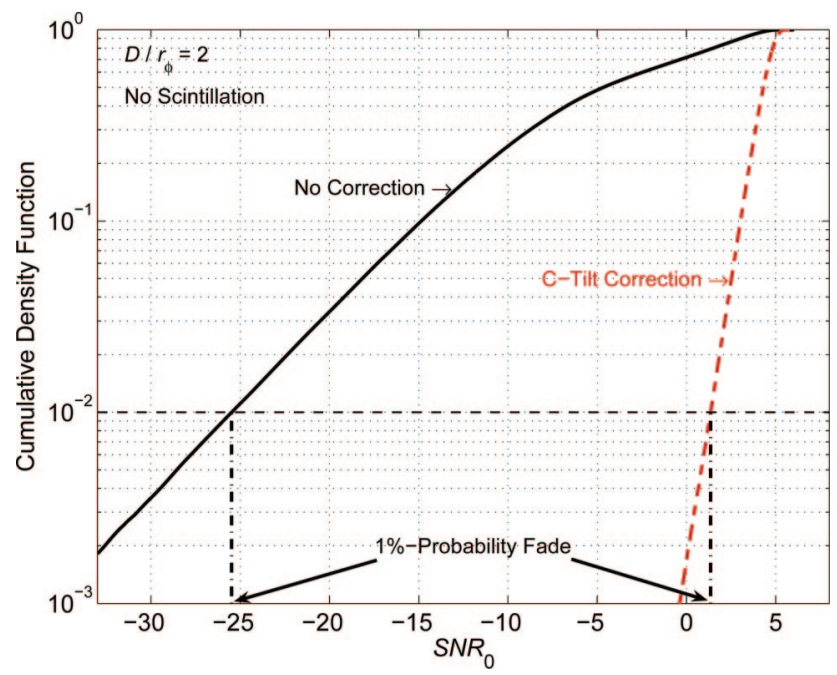

Fig. 4. (Color online) Cumulative density function of $\mathrm{SNR}_{0}$ for the case $D / r_{\phi}=2$.

function of $D / r_{\phi}$. Mean and variance are identical for $F_{i_{\phi}}$ and $F_{\text {beta. }}$. Thus moments of higher orders are here compared. At approximately $D / r_{\phi}=8$, with a maximum CDF difference less than $10^{-2}$, the beta fit can be considered good. For $D / r_{\phi}>10$, the fit worsens because the beta model does not tend to the Rayleigh distribution.

To study the strength of the $\mathrm{SNR}_{0}$ fades as a function of $D / r_{\phi}$, we consider the level under which $\mathrm{SNR}_{0}$ lies with a probability of $1 \%$. In Fig. 4 the CDF of $\mathrm{SNR}_{0}$ is plotted for the particular case $D / r_{\phi}=2$ and the $1 \%$-probability fade level is indicated. Figure 5 shows the $1 \%$-probability fade of $\mathrm{SNR}_{0}$ as a function of $D / r_{\phi}$. We note that, at $1 \%$ probability, the SNR starts to decrease when $\sigma_{i, 0}{ }^{2}$ starts a sharp increase (compare with Fig. 2). Interestingly, whereas $D / r_{\phi}$ $\approx 2$ represents a maximum for the fade level of the tilt-corrected wave, it is a local minimum for that of the uncorrected wave. For $D / r_{\phi} \rightarrow \infty$, the fade level

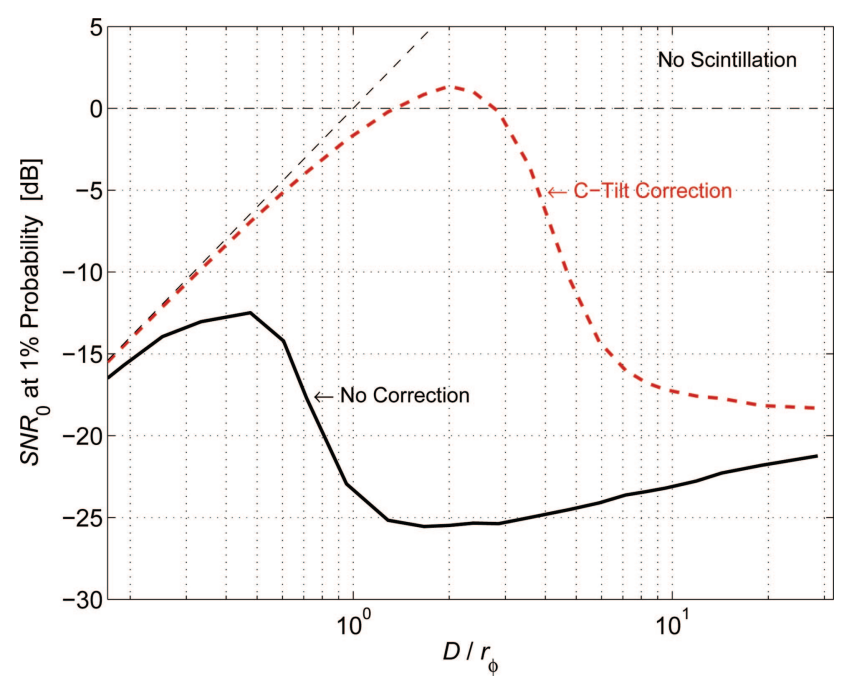

Fig. 5. (Color online) Level of fades occurring with a probability of $1 \%$ for $i=i_{\phi}$. 
tends to $-18.9 \mathrm{~dB}$ as a result of the CLT. The $1 \%$ probability fade is here an expedient means of assessing a link. Because most bit errors occur during $1 \%$-probability fades, the variations of the mean biterror rate (in particular, variations with respect to the aperture size) inversely follow the variations of these fades. Also relevant is the depth of the fades with respect to the mean $\left\langle\mathrm{SNR}_{0}\right\rangle$. These fades, which are supposed to occur $1 \%$ of the time, are known to be much longer than a bit duration. Error bursts associated with deep fades are then difficultly mitigated by channel coding.

We observe in Figs. 2 and 5 a transition regime where the fluctuations strongly increase. This transition regime occurs at approximately $D / r_{\phi}=1$ for an uncorrected wave and at approximately $D / r_{\phi}=5$ for a tilt-corrected wave. Similar transition regimes have been found by Andrews et al. when evaluating scintillation for an uplink scenario [22]. In this case, the principle of reciprocity is applied with the parameter $D$ denoting the diameter of the transmitting aperture. In Fig. 10 of [22] the transition regimes for the scintillation index can be observed at approximately $D / r_{\phi}=1$ and $D / r_{\phi}=5$ for an uncorrected beam and a corrected beam, respectively.

\section{Phase and Amplitude Perturbations}

A. Intensity and Amplitude Statistics

Under weak fluctuations, amplitude and intensity fields are generally lognormally distributed [13]. Intensity, amplitude, and log-amplitude $\chi$ are related by $I=A^{2}=\exp (2 \chi)$. Knowing the spatial covariance function $B_{I}(\rho)$ of the lognormally distributed intensity, the spatial correlation function $R_{A}(\rho)$ of the amplitude is readily found from

$$
R_{A}(\rho)=\left[\frac{B_{I}(\rho)+1}{\sigma_{I}^{2}+1}\right]^{1 / 4} .
$$

$R_{A}(\rho)$ is related to the log-amplitude structure function $D_{x}(\rho)$ by

$$
R_{A}(\rho)=\exp \left[-0.5 D_{\chi}(\rho)\right] .
$$

From Eq. (17) we deduce that the mean amplitude is given by

$$
\langle A\rangle=\left(\sigma_{I}^{2}+1\right)^{-1 / 8} .
$$

We will consider a spatial power spectrum $F_{I}(\kappa)$ of the intensity based on that predicted by the Rytov theory for a plane wave propagating through turbulence of constant strength and characterized by a Kolmogorov spectrum. We thus have [11]:

$$
F_{I}(\kappa) \propto\left[1-\frac{\kappa_{I}^{2}}{\kappa^{2}} \sin \left(\frac{\kappa^{2}}{\kappa_{I}^{2}}\right)\right] \kappa^{-11 / 3},
$$

where $\kappa_{I}$ is the characteristic spatial frequency of the intensity field. Defining the intensity correlation length $\rho_{I}$ as the $1 / e^{2}$ crossing point of the normalized intensity covariance function [1], we have $\rho_{I} \approx$ $1.4 / \kappa_{I}$. The scaling of the spectrum $F_{I}(\kappa)$ is determined by $\sigma_{I}^{2}$.

\section{B. Phase-Amplitude Dependence}

Because amplitude fluctuations arise from phase fluctuations, a dependence between the transverse phase and amplitude fields must be present. Even though the mean square photocurrent $\left\langle i^{2}\right\rangle$ is found not to be strongly affected by this dependence [3], other moments may be. It is, however, generally difficult to characterize this dependence for propagations through turbulent media. How often does an area of almost constant amplitude ride an area of almost constant phase? The Rytov theory allows the evaluation of the correlation function $\Re_{x \phi}(\rho)$ between phase and amplitude fluctuations (see [11], p. 235). This correlation function is defined by

$$
\Re_{\chi \phi}(\rho) \equiv \frac{D_{\chi \phi}(\rho)}{\sqrt{D_{\chi}(\rho) D_{\phi}(\rho)}},
$$

with $D_{\chi \phi}(\rho)$ being the cross-structure function of $\chi$ and $\phi$. Tatarskii [11] shows that $\Re_{\chi \phi}(0)$ is significantly less than $1\left(\Re_{\chi \phi}(0) \approx 0.33\right)$ for the case $\sqrt{L / k} \ll l_{0}$ where $k$ is the wavenumber, $L$ is the propagation distance, and $l_{0}$ is the inner scale of turbulence, with a constant turbulence strength over the path. For the more important case $\sqrt{L / k} \gg l_{0}$, it was found that $R_{\chi \phi}(0) \ll 1$. So, for constant turbulence strength, the correlation decreases as the propagation distance increases. Assuming field statistics as given by the Rytov theory, numerical realizations of amplitude and phase fields containing a cross correlation can be generated [23]. Investigating phase and amplitude correlation, other authors [24] have observed from a numerical propagation simulation that the average phase gradient at the center of a speckle spot (i.e., a spot of high amplitude) was less than $1 / 3$ the average phase gradient of the whole amplitude field; unfortunately, the physical parameters of the simulated scenario are not provided.

In Subsections 4.C-4.E we look at a scintillation index of 2. Considering the fact that amplitude fluctuations require a given propagation through the turbulent medium to develop and strengthen whereas phase fluctuations do not, we easily imagine propagation scenarios where phase and amplitude fluctuations are highly uncorrelated. In the following, we assume amplitude and phase independent in the aperture plane.

\section{Photocurrent Approximation}

To use the simulation results obtained for $i_{\phi}$, we approximate the statistics of $i$ by the statistics of $i_{A} i_{\phi}$ where

$$
i_{A} \equiv \int W(\mathbf{r}) A(\mathbf{r}) \mathrm{d} \mathbf{r} .
$$

Considering $i_{A} i_{\phi}$, the $\mathrm{C}$-tilt correction is performed on just $i_{\phi}$. The behavior of $i$ for limit cases is given in 


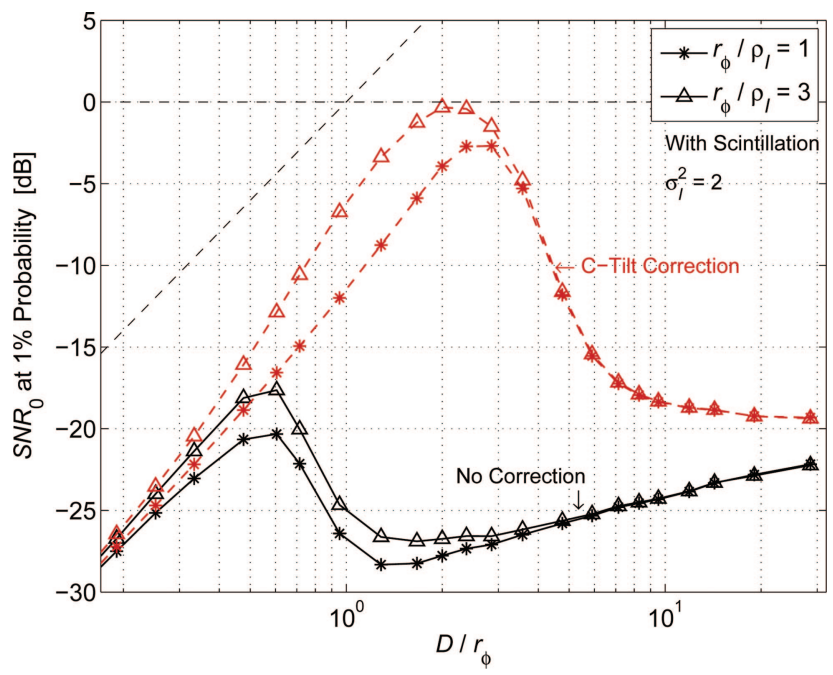

Fig. 8. (Color online) Same as Fig. 5 but with scintillation.

$$
F_{i}(x)=\int_{0}^{1} f_{i_{\phi}}(y) F_{i_{A}}\left(\frac{x}{y}\right) \mathrm{d} y,
$$

where $f_{i_{\phi}}$ is the probability density function of $i_{\phi}$ and $F_{i_{A}}$ is the CDF of $i_{A}$. Like $A, i_{A}$ is taken as a lognormal variable.

\section{Signal-to-Noise Fading}

Scintillation does not affect strongly the mean SNR. Assuming $i \approx i_{A} i_{\phi},\left\langle\mathrm{SNR}_{0}\right\rangle$ is proportional to $\left\langle i_{A}{ }^{2}\right\rangle$, which, as $D$ increases, decreases from 1 and saturates at $\langle A\rangle^{2}$. For $\sigma_{I}^{2}=2$, we have $\langle A\rangle^{2} \approx 0.76$, which is negligible compared to fade depths. Figure 8 shows, in presence of scintillation, the level of the $1 \%$ probability fade for $\mathrm{SNR}_{0}$. Equation (28) was used to calculate the fade levels. Scintillation introduces deep fades at low $D / r_{\phi}$ values, that is, where wavefront distortions may be negligible. Note also that the optimum aperture size, as seen from Fig. 8, is close to that without scintillation (see Fig. 5).

\section{E. Comparison with Direct Detection}

We now wish to compare the impact of turbulence on a coherent system and on a direct-detection (DD) system. Direct detection suffers from scintillation as the signal photocurrent is proportional to the optical power detected. In a DD system, two noise types can be distinguished: a noise constant and independent of the received power, such as thermal noise, and shot noise, which increases with the received power. The signal-to-noise ratio $\mathrm{SNR}_{D D, T N}$ of a $\mathrm{DD}$ system limited to thermal noise is proportional to the square of the received power,

$$
\mathrm{SNR}_{D D, T N} \propto P^{2}
$$

with

$$
P=\int W(\mathbf{r}) A^{2}(\mathbf{r}) \mathrm{d} \mathbf{r} .
$$

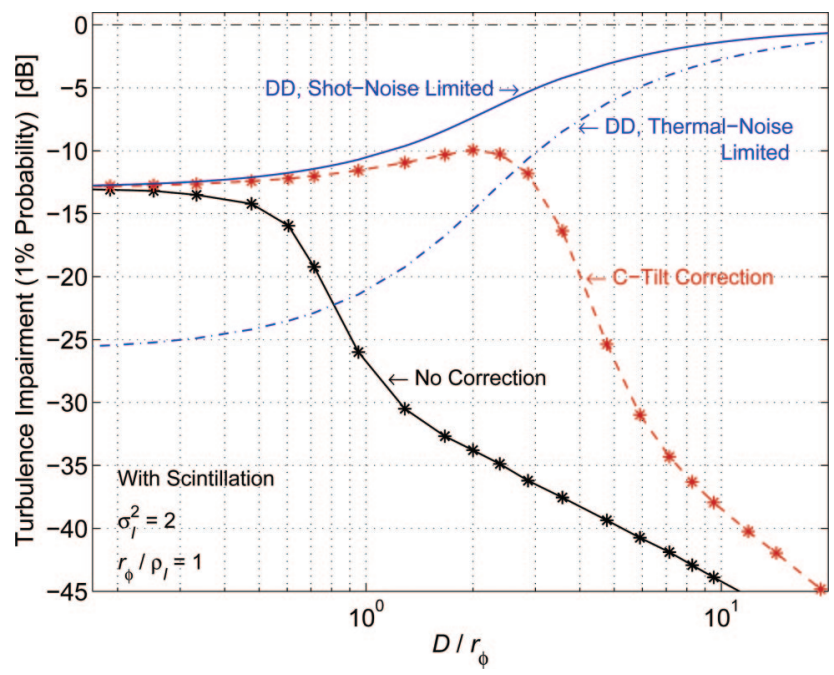

Fig. 9. (Color online) Impairment caused by turbulence on the SNR. Results for coherent and direct detections are displayed (DD $=$ direct detection).

When the receiver is limited by shot noise, we have:

$$
\mathrm{SNR}_{D D, S N} \propto P .
$$

For different types of receiver, Fig. 9 shows the impairment (loss) caused by turbulence on the SNR as compared to a vacuum propagation. The impairment is calculated as the 1\%-probability fade. The scintillation is defined here by $\sigma_{I}^{2}=2$ and $r_{\phi} / \rho_{I}$ $=1$. Like $i_{A}, P$ is assumed lognormally distributed. We see from Fig. 9 that, in the presence of turbulence, a coherent system can compete with a DD system only under the two following conditions: (i) the aperture size is limited, (ii) the DD counterpart suffers from significant thermal noise (or another signalindependent noise). For a coherent system to reach the loss curve of the DD shot-noise-limited system, an ideal adaptive-optics system would be required.

Now one may regard the DD impairment curves of Fig. 9 as somewhat optimistic. Indeed, wavefront distortions (equivalently, $r_{\phi}$ ) are not taken into account in the impairment calculation for the DD cases. To detect all the spatial modes contained in a distorted wavefront, it should be assured that the photodetector placed in the focal plane fully overlaps the expanded focal spot. Making the detector area larger to account for turbulence-induced focal spot expansion is detrimental in terms of collected background light, and detector noise and bandwidth. The choice of the detector size and its impact on the system performance can be a complex problem and is beyond the scope of this paper. However, it is of interest to quantify the necessary increase of detector size as wavefront distortions worsen. The average size of the focal spot is given by the point spread function (PSF). Andrews and Phillips provide approximations of the long-term PSF (no tilt correction) and short-term PSF (with tilt correction) [13]. Noting $\mathrm{PSF}_{L T}$ and $\mathrm{PSF}_{S T}$ the long- and short-term PSFs, respectively, we have 


$$
\begin{aligned}
& \operatorname{PSF}_{L T}(r) \sim \exp \left[-K \frac{D^{2}}{\left(1+D / r_{\phi}\right)^{5 / 3}} r^{2}\right] \\
& \operatorname{PSF}_{S T}(r) \sim \exp \left[-K \frac{D^{2}}{\left(1+0.28 D / r_{\phi}\right)^{5 / 3}} r^{2}\right] .
\end{aligned}
$$

$K$ is here a constant. In the absence of turbulence, the $\mathrm{PSF}$ approximation gives $\mathrm{PSF}_{0}(r) \sim \exp \left(-K D^{2} r^{2}\right)$. Let $\beta_{\text {det }}$ be the factor by which the detector diameter must be increased due to turbulence. We readily have

$$
\beta_{\operatorname{det}}=\left(1+D / r_{\phi}\right)^{5 / 6},
$$

when no correction is applied, and

$$
\beta_{\mathrm{det}}=\left(1+0.28 D / r_{\phi}\right)^{5 / 6},
$$

when the tilt is corrected. To give an impression of the impact of wavefront distortions on DD systems, Fig. 10 shows the aperture-averaging factor and the detector diameter factor $\beta_{\text {det }}$ on the same graph as a function of $D / r_{\phi}$. The aperture-averaging factor, noted $a$, is defined as the ratio of the variance of $P$ to the scintillation index $\sigma_{I}^{2}$ :

$$
a=\frac{\sigma_{P}^{2}}{\sigma_{I}^{2}}
$$

It is thus a measure for the reduction of scintillation fades. Curves of $a$ were evaluated for $r_{\phi} / \rho_{I}$ values of 1 and 3. The factor $\beta_{\text {det }}$ by which the detector size should be increased was evaluated for uncorrected and tilt-corrected waves. One sees that scintillation reduction through increased aperture size reaches a limit set by the maximum acceptable detector size. In the context of detrimental background light and high

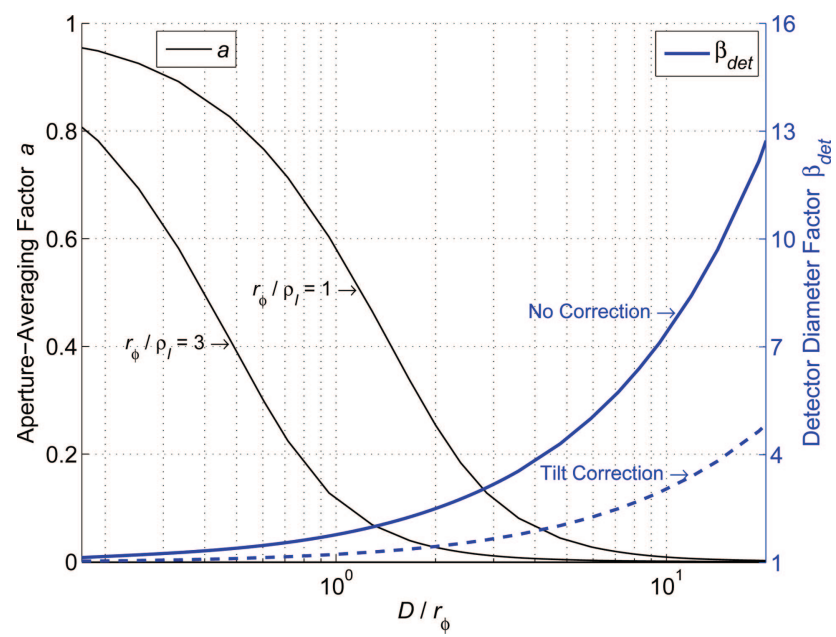

Fig. 10. (Color online) Aperture averaging factor $a$ and detector diameter factor $\beta_{\operatorname{det}}$ plotted on the same graph. As the receiver diameter $D$ increases less scintillation is experienced due to aperture averaging. But the size of the detector in the focal plane must be adjusted to account for growing wavefront distortions.
$D / r_{\phi}$ values, tilt correction is beneficial also for direct detection since it allows one to keep a smaller detector and thus to reduce the field of view.

\section{Conclusion}

In optical coherent communications through turbulence, the analysis of fading probability helps in choosing the correct receiving aperture size. Spatial fields based on Kolmogorov statistics of turbulence have been numerically generated and have provided distribution instances of the coherent signal. It was found that, without tilt correction, deleterious SNR fades are expected for $D / r_{\phi}>0.5$ (Fig. 5). When the tilt is corrected, such fades are expected for $D / r_{\phi}$ $>2$, which contrasts with the mean SNR climaxing at $D / r_{\phi} \approx 5$ (Fig. 1).

Scintillation was assumed independent of the phase perturbations and was characterized according to the weak-fluctuation theory. This may not be accurate with respect to what can be measured in the saturation regime [1,26,27]. An approximation was also introduced to study scintillation independently of wavefront distortions. Nevertheless, some useful assertions can be deduced regarding coherent receivers. Although scintillation can greatly impair the communication quality, it has little influence on the optimum aperture size (Fig. 8). The latter is predominantly determined by the ratio $D / r_{\phi}$. Aperture averaging of scintillation has, for an uncorrected wave, a limited positive impact because the associated fade reduction is perceivable only when $\rho_{I}<D<r_{\phi}$.

\section{References}

1. L. C. Andrews, R. L. Phillips, and C. Y. Hopen, Laser Beam Scintillation with Applications (SPIE, 2001).

2. J. C. Ricklin, S. Bucaille, and F. M. Davidson, "Performance loss factors for optical communication through clear air turbulence," Proc. SPIE 5160, 1-12 (2004).

3. D. L. Fried, "Optical heterodyne detection of an atmospherically distorted signal wave front," Proc. IEEE 55, 57-66 (1967).

4. A. R. Weeks, J. Xu, R. R. Phillips, L. C. Andrews, C. M. Stickley, G. Sellar, J. S. Stryjewski, and J. E. Harvey, "Experimental verification and theory for an eight-element multiple-aperture equal-gain coherent laser receiver for laser communications," Appl. Opt. 37, 4782-4788 (1998).

5. J. H. Churnside and C. M. McIntyre, "Signal current probability distribution for optical heterodyne receivers in the turbulent atmosphere. 1. Theory," Appl. Opt. 17, 2141-2147 (1978).

6. J. H. Churnside and C. M. McIntyre, "Signal current probability distribution for optical heterodyne receivers in the turbulent atmosphere. 2. Experiment," Appl. Opt. 17, 2148-2152 (1978).

7. K. A. Winick, "Atmospheric turbulence-induced signal fades on optical heterodyne communication links," Appl. Opt. 25, 18171825 (1986).

8. J. E. Kaufmann, "Performance limits of high-rate space-toground optical communications through the turbulent atmospheric channel," Proc. SPIE 2381, 171-182 (1995).

9. R. Lange, B. Smutny, B. Wandernoth, R. Czichy, and D. Giggenbach, "142 km, 5.625 Gbps free-space optical link based on homodyne BPSK modulation," Proc. SPIE 6105, 61050A (2006).

10. R. Lange and B. Smutny, "Homodyne BPSK-based optical in- 
tersatellite communication links," Proc. SPIE 6457, 645703 (2007).

11. V. I. Tatarskii, The Effects of the Turbulent Atmosphere on Wave Propagation (Israel Program for Scientific Translations, 1971).

12. J.-M. Conan, G. Rousset, and P.-Y. Madec, "Wave-front temporal spectra in high-resolution imaging through turbulence," J. Opt. Soc. Am. A 12, 1559-1570 (1995).

13. L. C. Andrews and R. Phillips, Laser Beam Propagation through Random Media, 2nd ed. (SPIE, 2005).

14. J. W. Strohbehn, "Line of sight wave propagation through the turbulent atmosphere," Proc. IEEE 56, 1301-1318 (1968).

15. D. L. Fried and J. L. Vaughn, "Branch cuts in the phase function,” Appl. Opt. 31, 2865-2882 (1992).

16. C. M. Harding, R. A. Johnston, and R. G. Lane, "Fast simulation of a Kolmogorov phase screen," Appl. Opt. 38, 2161-2170 (1999).

17. J. D. Barchers, D. L. Fried, and D. J. Link, "Evaluation of the performance of Hartmann sensors in strong scintillation," Appl. Opt. 41, 1012-1021 (2002).

18. A. Glindemann, "Relevant parameters for tip-tilt systems on large telescopes," Publ. Astron. Soc. Pac. 109, 682-687 (1997).

19. G.-M. Dai, "Modal compensation of atmospheric turbulence with the use of Zernike polynomials and Karhunen-Loeve functions," J. Opt. Soc. Am. A 12, 2182-2193 (1995).
20. A. Papoulis and S. U. Pillai, Probability, Random Variables, and Stochastic Processes, 4th ed. (McGraw-Hill, 2002).

21. L. C. Andrews, Special Functions of Mathematics for Engineers, 2nd ed. (SPIE Oxford Science Publications, 1998).

22. L. C. Andrews, R. L. Phillips, R. J. Sasiela, and R. R. Parenti, "Strehl ratio and scintillation theory for uplink Gaussianbeam waves: beam wander effects," Opt. Eng. 45, 076001 (2006).

23. D. Kouznetsov, V. V. Voitsekhovich, and R. Ortega-Martinez, "Simulations of turbulence-induced phase and log-amplitude distortions," Appl. Opt. 36, 464-469 (1997).

24. N. Shvartsman and I. Freund, "Speckle spots ride phase saddles sidesaddle," Opt. Commun. 117, 228-234 (1995).

25. B. L. McGlamery, "Computer simulation studies of compensation of turbulence degraded images," Proc. SPIE 74, 225-233 (1976).

26. J. H. Churnside and R. G. Frehlich, "Experimental evaluation of lognormally modulated Rician and IK models of optical scintillation in the atmosphere," J. Opt. Soc. Am. A 6, 1760-1766 (1989).

27. N. Perlot, D. Giggenbach, H. Henniger, J. Horwath, M. Knapek, and K. Zettl, "Measurements of the beam-wave fluctuations over a $142 \mathrm{~km}$ atmospheric path," Proc. SPIE 6304, 630410 (2006). 